\title{
Are different generations of CAD/CAM milling machines capable to produce restorations with similar quality?
}

\author{
Renato Roperto ${ }^{1}$, Hussein Assaf ${ }^{2}$, Thiago Soares-Porto ${ }^{2}$, Lisa Lang ${ }^{3}$, Sorin Teich ${ }^{4}$
}

${ }^{1}$ DDS, MS, PhD, Department of Comprehensive Care, School of Dental Medicine - Case Western Reserve University, 2124 Cornell Rd, Cleveland, 44106, USA

${ }^{2}$ DDS, MS, Department of Comprehensive Care, School of Dental Medicine - Case Western Reserve University, 2124 Cornell Rd, Cleveland, 44106, USA

${ }^{3}$ DDS, MS, MBA, Department of Comprehensive Care, School of Dental Medicine - Case Western Reserve University, 2124 Cornell Rd, Cleveland, 44106, USA

${ }^{4}$ DDS, MBA, Department of Comprehensive Care, School of Dental Medicine - Case Western Reserve University, 2124 Cornell Rd, Cleveland, 44106, USA

Correspondence:

Department of Comprehensive Care

School of Dental Medicine - Case Western Reserve University

2124 Cornell Rd

Cleveland, 44106, USA

rcr67@case.edu

\begin{abstract}
Roperto R, Assaf H, Soares-Porto T, Lang L, Teich S. Are different generations of CAD/CAM milling machines capable to produce restorations with similar quality? J Clin Exp Dent. 2016;8(4):e423-8.

http://www.medicinaoral.com/odo/volumenes/v8i4/jcedv8i4p423.pdf
\end{abstract}

Received: 19/01/2016 Accepted: 27/01/2016

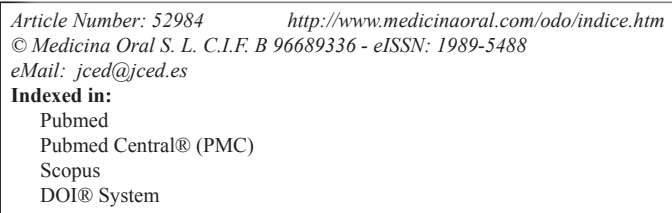

\begin{abstract}
Background: Different CAD/CAM machines' generation may impact the restoration overall quality. The present study evaluated the marginal fit of CAD/CAM restorations manufactured with different generations of CEREC milling unit systems.

Material and Methods: Sixteen typodont teeth were divided into two groups $(n=8)$ according to the machine's generation assigned. These are control group (G1): Cerec AC with Bluecam/Cerec 3 milling unit and (G2): Cerec AC with Bluecam/MC XL Premium Package milling unit. Scanning of the preparation were performed and crowns were milled using the Vita Mark II blocks. Blocks were cemented using epoxy glue on the pulpal floor only and finger pressure applied for $1 \mathrm{~min}$. Upon completion of the cementation step, misfits between the restoration and abutment were measured by microphotography and the silicone replica technique using light body silicon material on Mesial (M) and Distal (D) surfaces.

Results: Mean and SDs of marginal gaps in micrometers were: G1/M: $94.90( \pm 38.52)$, G1/D: $88.53( \pm 44.87)$, G2/M: 85.65 ( \pm 29.89$),$ G2/D: 95.28 ( \pm 28.13$)$. Two-way ANOVA indicated no significant differences among different groups $(P>0.05)$; surface area $(P>0.05)$ and the interaction $(P>0.05)$. Overall, $\mathrm{G} 2$ had greater margin gaps than G1, however, without statistical difference $(P>0.05)$.

Conclusions: Difference in milling unit generation did not significantly affect the marginal fit. Marginal gap means were in the range of the clinical acceptance levels for both generations of Cerec milling units, regardless the teeth site area.
\end{abstract}

Key words: $C A D / C A M$, margin, ceramics. 


\section{Introduction}

Cerec CAD/CAM machines are currently used to manufacture ceramic restorations based on computer-assisted design and produce a restoration on a single dental appointment. These restorations, commonly made with ceramic material, are becoming increasingly popular worldwide (1-4). Because the technology is costly, some clinicians are still using previous generations of CAD/ $\mathrm{CAM}$ equipment to fabricate and delivery intraoral restorations.

Margin quality has been described as one of the most important aspects when comes to longevity of CAD/ CAM ceramic restorations (5-12). Margin discrepancies beyond $100 \mu \mathrm{m}$ may impact the survival rate by causing microleakage, staining, tooth sensitivity, recurrent caries, periodontal problems, and ultimately failure of the entire restoration $(13,14)$.

The present study was designed to compare and evaluate the marginal fit of ceramic $\mathrm{CAD} / \mathrm{CAM}$ ceramic restorations produced with the Cerec AC using BlueCam and milled with different Cerec milling generations: i) Cerec 3 Milling unit (Cerec3) and ii) Cerec MC XL Premium Package (MCXLPP) milling unit. First, we determined whether the marginal fit would be affected by the different milling unit used, and second, we examined if the different regions of the tooth (mesial and distal) differ in terms of marginal fit, regardless of the milling unit used.

\section{Material and Methods}

This study was approved by an ethics committee. Sixteen maxillary typodonts (Kilgore typodont model 200 - Kilgore International, Coldwater, MI, USA) with unrestored and intact teeth were used in this study. The upper left second pre-molars typodont teeth received a full-contour crown preparation for Cerec with 6-80 degrees of axial wall conversion, $1.0 \mathrm{~mm}$ axial reduction, and a $2.0 \mathrm{~mm}$ flat occlusal reduction on both functional and non-functional cusps. A flat 90o shoulder margin design was used for all preparations.

The preparations were made using cone-shape diamond points (016 FG medium round end taper diamond) followed by a finishing diamond point with the same shape (Brasseler, Savannah, GA, USA). The gingival margin was then finished with a flat end diamond point (014 FG medium flat end taper diamond - Brasseler), resulting in a $1.0 \mathrm{~mm}$ width circumferential flat shoulder. All preparations had $0.5 \mathrm{~mm}$ supragingival margins and they were prepared by one investigator. A new set of diamond points was used for every four prepared teeth. Teeth were then randomly assigned to two groups $(n=8)$. These are control group (G1): Cerec 3 Milling unit (Cerec3) and ii) Cerec MC XL Premium Package milling unit (MCXLPP) (Fig. 1). Technical differences between these two machines are listed in table 1.

A Cerec AC unit with Bluecam (Sirona) equipped with Microsoft Windows 7-64 bits (Microsoft Corporation, Redmond, WA, USA) that run the Cerec software version 4.3 (Sirona) was used in this study. For each group of teeth, the intra-oral camera was calibrated using the Sirona camera calibration kit. An uniform layer of antireflective spray (Sirona Optspray) was sprayed on all teeth surface area, including the surrounding soft tissue area. Special care was taken to avoid over-powdering the prepped teeth and adjacent surfaces, which were then scanned with the intra-oral camera. Before generating the 3D model, scanned images for both G1 and G2 were evaluated on the computer screen using the "distance" software tool in order to confirm preparation metrics. A tri-dimensional (3D) model was then generated for each tooth; the adjacent teeth were digitally set in the virtual model axis and trimmed for proximal contact adjustments. Preparation margin was designed using the "automatic margin finder" tool followed by the insertion axis adjustment. The Cerec software then generated the 3D proposal restoration using the following software crown parameters: spacer $=100 \mu \mathrm{m}$; occlusal milling offset $=0 \mu \mathrm{m}$; proximal contact strength $=50 \mu \mathrm{m}$; occlusal contact strength $=-75 \mu \mathrm{m}$; dynamic contact strength $=25 \mu \mathrm{m}$; minimal thickness (radial) $=0 \mu \mathrm{m}$; minimal thickness $($ occlusal $)=0 \mu \mathrm{m}$; margin thickness $=70 \mu \mathrm{m}$. After generating the $3 \mathrm{D}$ virtual restorations, scanned

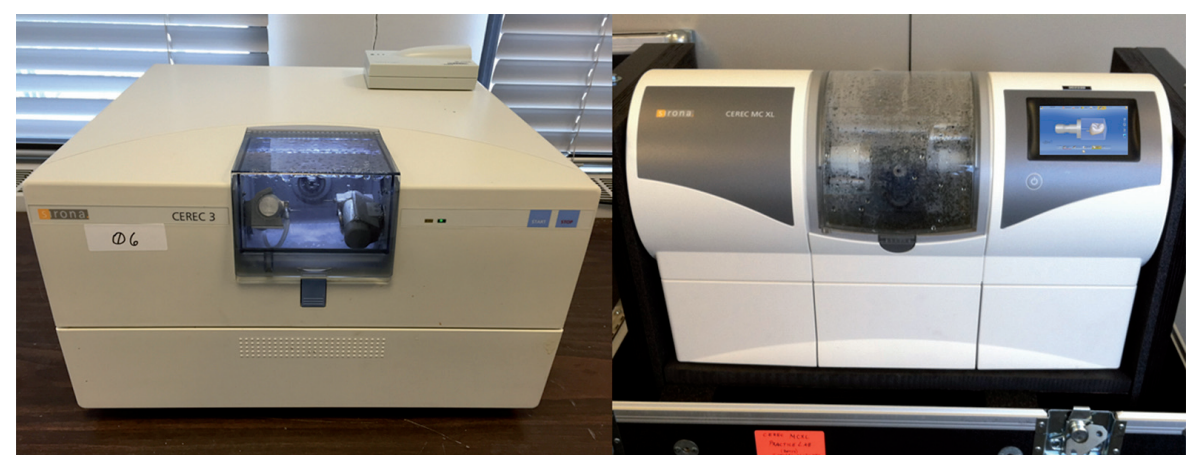

Fig. 1. Milling CAD/CAM units used in this study: Cerec 3 (left) and Cerec MXCL Premium Package (right). 
Table 1. Cerec 3 and Cerec MCXL Premium Package comparative chart.

\begin{tabular}{|c|c|c|}
\hline & Cerec 3 & Cerec MC XL Premium Package \\
\hline Indication & $\begin{array}{l}\text { Inlays, Onlays, veneers } \\
\text { and single crowns }\end{array}$ & $\begin{array}{l}\text { Inlays, Onlays, veneers, single crowns } \\
\text { and frameworks up to } 12 \text { units }\end{array}$ \\
\hline Milling accuracy & $\pm 25 \mu \mathrm{m}$ & $\pm 25 \mu \mathrm{m}$ \\
\hline Max. block size & $15,5 \times 19 \times 20 \mathrm{~mm}$ & $22 \times 40 \times 85 \mathrm{~mm}$ \\
\hline Diamond points RPM & $40.000 \mathrm{rpm}$ & $42.000 \mathrm{rpm}$ \\
\hline Milling speed & $0.4-0.6 \mathrm{~mm} / \mathrm{min}$ & $1.0-1.5 \mathrm{~mm} / \mathrm{min}$ \\
\hline Motors & 2 stepping motors & $\begin{array}{l}4 \text { stepping motors (not working } \\
\text { at the same time) }\end{array}$ \\
\hline Axes & 4-axis & 4-axis \\
\hline
\end{tabular}

images for both G1 and G2 were again evaluated on the computer screen using the "restoration thickness" tool in order to confirm restoration metrics.

The Cerec3 milling unit was calibrated using the Sirona Milling Calibration kit and a new set of diamond burs kit (step bur 12 and point bur 10) were used. One brand new water tank filled with distilled water and lubricant (Dentatec-Sirona) was used for the millings on this group. The MCXLPP was also calibrated by replacing the milling diamonds for the calibration phantom and pins. A brand new water tank with a brand new filter was used with distilled water mixed with lubricant (Dentatec-Sirona). The CAD/CAM feldsphatic ceramic blocks
Vita Mark II (Vita, Yorba Linda, CA, USA) size I12 and 1M1 shade ceramic blocks were employed to mill all the restorations for both groups. Upon completion of each milling process, blocks were replaced inside the milling chamber and the diamond burs inspected for damage or breakage. Prepared teeth were then stored in small envelopes with their respective ceramic restorations according to the group assigned. The sequence for tooth scanning, crown design and milling preview is shown in figure 2 .

Teeth were then submitted to the micrographic silicon replica technique for margin misfit evaluation. The silicon replica technique flow chart is shown in figure 3.

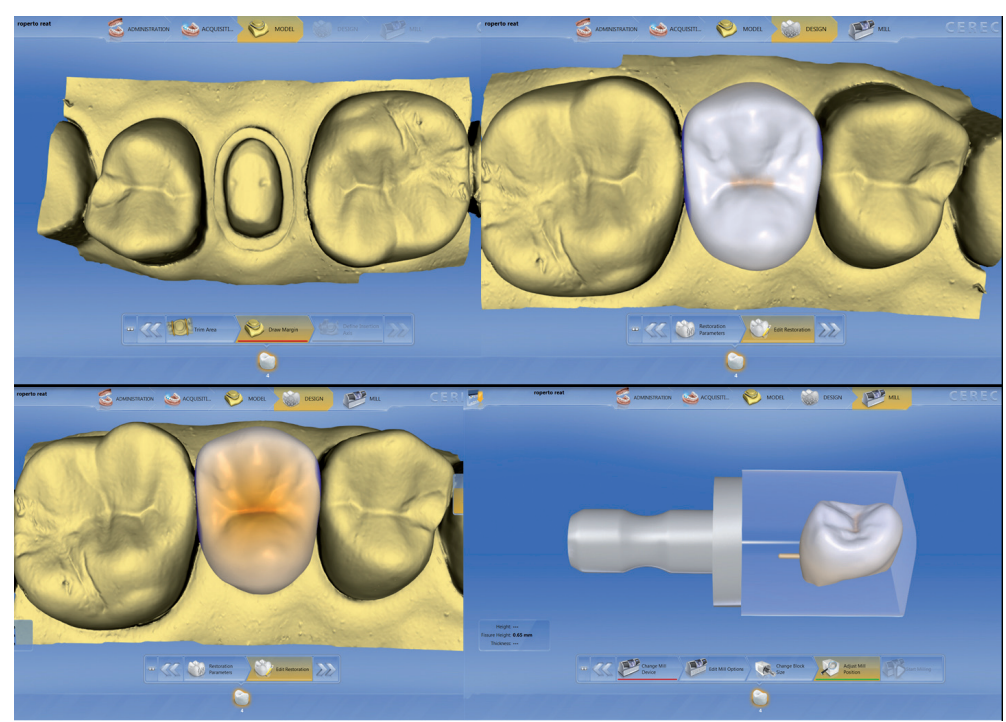

Fig. 2. Sequence for tooth scanning (top left), crown design (top right), crown metrics adjustment (bottom left) and milling preview (bottom right). 


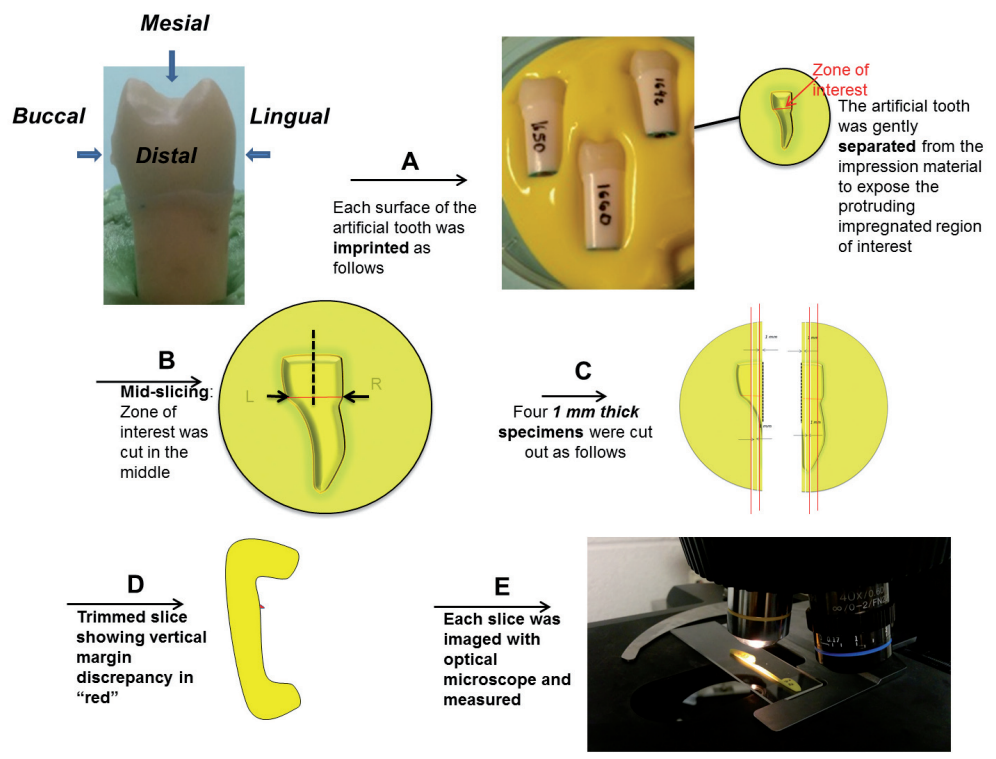

Fig. 3. Silicon replica technique sequence.

Mesial and distal surfaces of each group were evaluated separately as follows. The light body silicon based polymer was pooled in a shallow container. The tooth specimen was gently positioned into the uncured, low viscosity polymeric mixture without full submergence and allowed to remain inside the polymer until the substrate was fully cured. Subsequently, the tooth was gently separated from the polymeric material in order to expose impression of the region of interest. The polymeric imprint of a particular side of the tooth was cut in the middle with a fresh razor blade and subsequently 1 $\mathrm{mm}$ thick slices were cut from the specimen to the right and to the left of the mid-section, yielding overall eight slices for analysis. Each slice was carefully marked to identify its position and viewed in a reflective optical 40x magnification microscope (Nikon SMZ445, Melville, NY, USA) to measure the variations in the marginal fit. A linear glass scale with $10 \mu \mathrm{m}$ resolution was used to identify the measured length in the specimens.

Data were analyzed by statistical software (SPSS 23.0, IBM Software, Armonk, NY, USA). Differences in the milling units among groups, also the individual sites, were analyzed by two-way ANOVA with Tukey's post hoc test comparisons to find any statistically significant differences. The significance level was $\alpha=0.05$.

\section{Results}

Inspection of histograms, normal Q-Q plots, and box plots showed that the measurements for preparation type and each site individually are normally distributed. The mean and standard deviation (SD) associated with vertical gap $(\mu \mathrm{m})$ related to different milling units are: Cerec3 milling 91.71 \pm 41.61 and MCXLPP 90.46 \pm 29.20 . Among the sites; the two-way ANOVA analysis did not show statistical differences between both milling units (Table 2).
For G1 and G2, the analysis associated with individual sites did not show statistically significant differences $(P<0.05)$. For each milling unit generation, one-way analysis with Tukey's post hoc test was made individually to find where the differences among the sites occurred. The $p$ value was set at a 0.05 level of significance and is shown in table 3 , which is the 2-way ANOVA.

Table 2. Distribution of mean, standard deviation (SD) and variance for different machines and sites.

\begin{tabular}{|l|c|}
\hline & Mean (SD)* \\
\hline Cerec3 Machine Mesial & $85.65( \pm 29.89)$ \\
\hline Cerec3 Machine Distal & $95.28( \pm 28.13)$ \\
\hline MCXLPP Mesial & $94.90( \pm 38.52)$ \\
\hline MCXLPP Distal & $88.53( \pm 44.87)$ \\
\hline
\end{tabular}

*values are given in $\mu \mathrm{m}$.

\section{Discussion}

In the present study, the first null hypothesis was that margin discrepancy of CAD/CAM restorations designed by the same scan unit would be affected by different milling unit generations. For the second null hypothesis, we examined if the different margin sites of the tooth (mesial, and distal) differ in terms of fit, regardless of the milling units used.

We rejected the first null hypothesis because different milling unit generations end up exhibiting similar margin adaptation. Also, we reject the second null hypothesis because different sites on the teeth did not in fact influence the margin adaptation.

The most remarkable findings in the data collected were 
Table 3. Analyses of Variance (2-way ANOVA).

\begin{tabular}{|l|c|c|c|c|c|}
\hline Source & Type III Sum of Squares & df & Mean Square & F & Sig. \\
\hline Corrected Model & $2182.500^{\mathrm{a}}$ & 3 & 727.500 & .561 & .641 \\
Intercept & 1062153.125 & 1 & 1062153.125 & 819.663 & .000 \\
Machine & 50.000 & 1 & 50.000 & .039 & .845 \\
Site & 84.500 & 1 & 84.500 & .065 & .799 \\
Machine * Site & 2048.000 & 1 & 2048.000 & 1.580 & .211 \\
Error & 160684.375 & 124 & 1295.842 & & \\
Total & 1225020.000 & 128 & & & \\
Corrected Total & 162866.875 & 127 & & & \\
\hline
\end{tabular}

the similarity in accuracy from both generations of CAD/CAM milling units. During the past three decades, enormous hardware and software improvement on the chair-side acquisition CAD/CAM units have been made. Not differently, the milling units have changed dramatically with main improvements towards speed, noise and better connectivity interface.

The Cerec MC XL premium package is faster and quitter and, it is the current Sirona's flagship model for chairside restorations. The Cerec 3 milling unit is currently discontinued, however, still largely used by clinicians because its robust technology, ability to connect to most of the modern Cerec acquisition units, and easy and cheap to repair when maintenance is necessary.

According to Shim et al. (15), a fit of a CAD/CAM restoration can be affected by different parameters settings and different software versions. These authors compared the Cerec software 3.8 and 4.2 versions and found that different generation of software can affect the overall quality of the restoration. This study used a Cerec AC with Bluecam unit equipped with the Cerec 4.3 version once this combination has been documented to be a reliable and highly accurate 3-D scan system. Bosh et al. (16) used the same scan system in combination with different milling units and concluded that 5-axis milling units presented better overall quality compared with 4-axis machines. Hamza et al. (17) found that 5-axis milling unit can actually improve productivity and precision by using the machine's additional axis. Contrarily, Cho et al. (18) concluded that the quality of the final CAD/CAM restoration does not increase with the number of steps and/or bur axes, instead, depending to a greater extent on digitalization, data processing, and production process. The limitation of chair-side CAD/ CAM machines containing no more than 4-axis make it difficult to compare this study with ours.

We also observed that distal surfaces of teeth had greater margin discrepancy than the mesial surface. This can be explained by the fact that distal surface of teeth was more difficult to be prepared under indirect vision. Because the mesial surfaces could be easily visualized by direct access, better margin quality was obtained during the preparation phase.

This study did not use natural human extracted teeth because the authors wanted to avoid a large variation due to age, individual structure, and storage time after extraction; consequently, typodont teeth provided a more uniform and standardized abutment. It was also not the objective of this study to test adhesion of teeth to CAD/ CAM restorations; instead, crowns were not cemented to the typodont teeth, therefore allowing direct viewing and external measurement. The vertical cervical marginal gap measurement was selected because it is the most common way to quantify the accuracy of fit $(15,17)$.

Using different typodont teeth instead of a single metal die was the selected method in order to introduce variability into the study. This research was not designed to compare different operators; preparations made by a single investigator better simulated a clinical environment and real application of CAD/CAM procedures. Future in-vitro and in-vivo research in margin discrepancy for chair-side CAD/CAM restorations should be conducted, comparing different brands of available machines with different generations of software and hardware, as well as different CAD/CAM block materials.

Within the limitations of this in vitro study, it was concluded that similar margin accuracy was found for both Cerec3 and MCXLPP machines. It seems likely that different generation of Cerec milling units are able to produce clinically acceptable restorations when used in combination with the Cerec BlueCam AC system.

\section{References}

1. Reich S, Schierz O. Chair-side generated posterior lithium disilicate crowns after 4 years. Clin Oral Investig. 2013;17:1765-72.

2. Mörmann W, Wolf D, Ender A, Bindl A, Göhring T, Attin T. Effect of Two Self-Adhesive Cements on Marginal Adaptation and Strength of Esthetic Ceramic CAD/CAM Molar Crowns. J Prosthodont. 2009;18:403-10.

3. Estafan D, Dussetschleger F, Agosta C, Reich S. Scanning electron microscope evaluation of CEREC II and CEREC III inlays. Gen Dent. 2003;51:450-4.

4. Bindl A, Mörmann WH. Clinical and SEM evaluation of all-ceramic chair-side CAD/CAM-generated partial crowns. Eur J Oral Sci. 2003;111:163-9. 
5. Yao J, Li J, Wang Y, Huang H. Comparison of the flexural strength and marginal accuracy of traditional and CAD/CAM interim materials before and after thermal cycling. J Prosthet Dent. 2014;112:649-57.

6. Giannetopoulos S, van Noort R, Tsitrou E. Evaluation of the marginal integrity of ceramic copings with different marginal angles using two different CAD/CAM systems. J Dent. 2010;38:980-6.

7. Souza RO, Özcan M, Pavanelli CA, Buso L, Lombardo GH, Michida SM, et al. Marginal and internal discrepancies related to margin design of ceramic crowns fabricated by a CAD/CAM system. J Prosthodont. 2012;21:94-100.

8. Tsitrou EA, Northeast SE, van Noort R. Evaluation of the marginal fit of three margin designs of resin composite crowns using CAD/ CAM. J Dent. 2007;35:68-73.

9. Akbar JH, Petrie CS, Walker MP, Williams K, Eick JD. Marginal adaptation of Cerec $3 \mathrm{CAD} / \mathrm{CAM}$ composite crowns using two different finish line preparation designs. J Prosthodont. 2006;15:155-63.

10. Mou SH, Chai T, Wang JS, Shiau YY. Influence of different convergence angles and tooth preparation heights on the internal adaptation of Cerec crowns. J Prosthet Dent. 2002;87:248-55.

11. Kim JH, Cho BH, Lee CH, Know SJ, Yi YA, Shin Y, et al. Influence of preparation design on fit and ceramic thickness of CEREC 3 partial ceramic crowns after cementation. Acta Odont Scandinavia. 2015;73:107-13.

12. Kassem AS, Atta O, El-Mowafy O. Fatigue resistance and microleakage of CAD/CAM ceramic and composite molar crowns. J Prosthodont. 2012;21:28-32.

13. Sturdevant JR, Bayne SC, Heymann HO. Margin gap size of ceramic inlays using second generation $\mathrm{CAD} / \mathrm{CAM}$ equipment. J Esthet Dent. 1999;11:206-14.

14. Krejci I, Lutz F, Reimer M. Marginal adaptation and fit of adhesive ceramic inlays. J Dent. 1993;21:39-46.

15. Shim JS, Lee JS, Lee JY, Choi YJ, Shin SW, Ryu JJ. Effect of software version and parameter settings on the marginal and internal adaptation of crowns fabricated with the CAD/CAM system. J Appl Oral Sci. 2015; 23:515-22.

16. Bosh G, Ender A, Mehl A. A 3-Dimensional accuracy analysis of chairside CAD/CAM milling processes. J Prosthet Dent. 2014;112:1425-31.

17. Hamza TA, Ezzat, HA, EL-Hossary MMK, Katamish HAEM, Shokry TE, Rosentiel SF. Accuracy of ceramic restorations made with two CAD/CAM systems. J Prosthe Dent. 2013;109:83-7.

18. Cho HD, Jun YT, Yang MY. Five axis CNC milling for effective machining of sculptured surfaces. Int J Prod Res. 1993;31:2559-73.

Acknowledgements

Dr. Bernard Tandler, Case Western Reserve University, provided editorial assistance.

\section{Conflict of Interest}

We state that all authors don't have any financial and personal relationships with other people or organizations that could inappropriately influence (bias) this work. We also state that neither employment, consultancies, stock ownership, honoraria, paid expert testimony, patents or patent applications, travel grants, all within 3 years of beginning the work submission were involved in this study. 